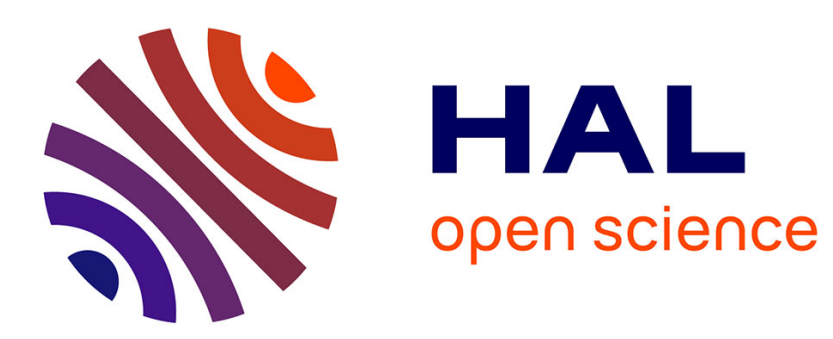

\title{
Parataxe apparente et coordination des interrogatifs en tchèque
}

Hana Skrabalova

\section{To cite this version:}

Hana Skrabalova. Parataxe apparente et coordination des interrogatifs en tchèque. Faits de langues, 2006, Coordination et subordination : typologie et modélisation (28), pp.231-242. hal-00599016

\section{HAL Id: hal-00599016 https://hal.science/hal-00599016}

Submitted on 8 Jun 2011

HAL is a multi-disciplinary open access archive for the deposit and dissemination of scientific research documents, whether they are published or not. The documents may come from teaching and research institutions in France or abroad, or from public or private research centers.
L'archive ouverte pluridisciplinaire HAL, est destinée au dépôt et à la diffusion de documents scientifiques de niveau recherche, publiés ou non, émanant des établissements d'enseignement et de recherche français ou étrangers, des laboratoires publics ou privés. 


\title{
Parataxe apparente et coordination des interrogatifs en tchèque
}

\author{
Hana Skrabalova*
}

\section{TROIS TYPES DE QUESTIONS A DEUX INTERROGATIFS EN TCHEQUE}

Dans cet article, nous proposons une analyse syntaxique de trois types de questions à deux interrogatifs en tchèque.

Dans le premier type de questions, que nous appelons questions multiples ${ }^{1,2}$, les deux mots interrogatifs sont préposés en début de phrase, $\mathrm{cf} .(1){ }^{3}$
(1)

$$
\begin{aligned}
& \text { Koho kde viděl ? } \\
& \text { qui-ACC où (a-t-il) vu }
\end{aligned}
$$$$
\text { (Q multiple) }
$$

Dans le deuxième type de questions, que nous appelons questions avec coordination de type 1 , les deux interrogatifs sont coordonnés par la conjonction $a$ ('et') et préposés en début de phrase, cf. (2a). Si la conjonction en (2a) est supprimée, nous obtenons une question multiple, cf. (2b). Ce fait suggère, de prime abord, que les interrogatifs dans les questions multiples constituent un cas de parataxe (c'est-à-dire une coordination sans conjonction visible). Dans cette perspective, la seule différence entre les questions en (2a) et (2b) est la

* CNRS, UMR 7110 (Laboratoire de linguistique formelle-LLF). E-mail: hana.skrabal @linguist.jussieu.fr.

${ }^{1}$ Cf. Comorowski (1986) pour le roumain, Rudin (1988) pour le bulgare, Lipták (2001) pour le hongrois.

${ }^{2}$ Les questions 'multiples' peuvent contenir plus de deux mots interrogatifs, cf. (i) et (ii). Elle ne contiennent pas pour autant plusieurs interrogations indépendantes, car tous les interrogatifs dénotent des entités participant à un seul événement.
(i) Kdo koho kde viděl ?
(ii) Kdo koho kde kdy viděl ?
qui-NOM qui-ACC où (a) vu qui-NOM qui-ACC où quand $(\mathrm{a}) \mathrm{vu}$

${ }^{3}$ Abréviations utilisées dans les gloses : ACC $=$ cas accusatif, AdvP = syntagme adverbial, $\mathrm{CL}=$ mot clitique, $\mathrm{COMP}=$ complémenteur $/$ conjonction de subordination, Conj = conjonction, $\mathrm{CoP}=$ syntagme de conjonction, $\mathrm{CP}=$ syntagme de complémenteur, $\mathrm{DAT}=$ cas datif, FinP = syntagme de finitude, ForceP $=$ syntagme de force phrastique, Int $=$ mot interrogatif, INSTR $=$ cas instrumental, $\mathrm{NOM}=$ cas nominatif, $\mathrm{NP}=$ syntagme nominal, $\mathrm{OBJ}$ $=$ objet, pro $=$ pronom nul, $\mathrm{Q}=$ question, $\mathrm{QUANT}=$ suffixe de quantification, $\mathrm{REFL}=$ pronom réfléchi, SUJ $=$ sujet, $t=$ trace, $\mathrm{TP}=$ syntagme de temps, $\mathrm{V}=$ verbe. 
réalisation phonétique d'une conjonction. Nous montrerons cependant plus loin (section 4) que cette hypothèse est incorrecte.
(2) a. Koho a kde
qui-ACC et où (a-t-il) vu
b. Koho kde viděl ?
qui-ACC où $(\mathrm{a}-\mathrm{t}-\mathrm{il}) \mathrm{vu}$
(coordination de type 1)

La coordination en (2a) est non-standard, car les interrogatifs ont chacun une fonction grammaticale différente (objet, circonstant) ${ }^{4}$. De telles coordinations ne sont pas en général possibles, cf. (3a) ${ }^{5}$. La coordination en (2a) est donc une coordination particulière qui diffère des coordinations attendues dans lesquelles les interrogatifs ont la même fonction grammaticale, comme en (3b).

(3) a. Viděl Petra (*a) v knihovně.

$\mathrm{vu}$ Pierre-ACC (et) en bibliothèque

'Il a vu Pierre à la bibliothèque.'

b. Koho a čeho se bojí?

qui-GEN et quoi-GEN REFL craint(-il)

'Qui et quoi craint-il ?

Enfin, dans le troisième type de questions, que nous appelons questions avec coordination de type 2, l'un des interrogatifs est préposé en début de phrase et l'autre est introduit par la conjonction $a$ ('et') en fin de la phrase, cf. (4a). (La séquence Conj $\mathrm{Int}_{2}$ peut être détachée et déplacée, voir section 5.2). La suppression de la conjonction en (4a) rend la phrase inacceptable, cf. (4b).

$$
\begin{aligned}
& \text { a. Koho viděl a kde ? } \\
& \text { qui-ACC (a-t-il) vu et où } \\
& \text { b. }{ }^{*} \text { Koho viděl kde? } \\
& \text { qui-ACC (a-t-il) vu où }
\end{aligned}
$$

(coordination de type 2)

L'article s'organise comme suit. Les sections 2 et 3 montrent que les questions présentées ci-dessus n'ont pas les mêmes propriétés sémantiques et syntaxiques. La section 4 présente des arguments contre la parataxe des interrogatifs dans les questions multiples. Enfin, la section 5 analyse la structure syntaxique de ces constructions. Nous défendons l'idée que chaque type de questions a une structure différente : (i) dans les questions multiples, les interrogatifs sont des constituants indépendants, (ii) dans les questions avec coordination de type 1 , les interrogatifs forment un constituant coordonné unique et (iii) les questions avec coordination de type 2 résultent d'une coordination phrastique ou d'une adjonction de la séquence Conj Int $_{2}$ à une question simple.

${ }^{4}$ Cf. Pullum et Zwicky (1986), Dik (1997), Peterson (2004).

$5 \mathrm{La}$ phrase (3a) devient meilleure avec un décrochement prosodique devant la conjonction (qui indique une structure incidente, cf. Abeillé 2003), ou bien lorsque les deux conjoints sont focalisés (voir section 5.4). 


\section{L'INTERPRETATION DES QUESTIONS A DEUX INTERROGATIFS}

Les questions multiples et les questions avec coordinations diffèrent du point de vue de leur interprétation. Les questions multiples reçoivent une interprétation de liste de paires, comme le montrent la paraphrase et la réponse en $(5)^{6}$.
a. Kdo co prinesl ?
qui-NOM quoi-ACC (a) apporté
b. pour chaque $\mathrm{x}, \mathrm{x}=$ personne, $\mathrm{x}$ a apporté quoi ?
c. Marie prrinesla salát, Petr zmrzlinu a Agáta ovoce.
Marie (a) apporté salade-ACC, Pierre glace-ACC et Agathe fruits-ACC

Les questions avec coordination de type I reçoivent, au contraire, une interprétation de paire spécifique, comme le montre l'exemple (6).

(6)
a. Kdo a co
přinesl ?
qui-NOM et quoi-ACC (a) apporté
b. pour quel $\mathrm{x}$ et $\mathrm{y}$ est-il vrai que $\mathrm{x}$ a apporté $\mathrm{y}$ ?
c. Marie přinesla koláč.
Marie (a) apporté gâteau-ACC

Les questions avec coordination de type 2 reçoivent également une interprétation spécifique, mais le premier interrogatif peut dénoter soit un seul individu, cf. $(7 \mathrm{~b}, \mathrm{c})$, soit un ensemble d'individus, cf. (7b', 7c'). Le deuxième interrogatif dénote toujours un seul élément (par exemple une indication temporelle) qui s'applique à cet individu ou à cet ensemble d'individus.

Par ailleurs, les questions avec coordination de type 2 peuvent être paraphrasées par une coordination de deux phrases interrogatives, cf. (8).

(7)

a. Kdo prišel a kdy ?
qui-NOM (est) venu et quand

b. pour quel $\mathrm{x}$ est-il vrai que $\mathrm{x}$ est venu et quand $\mathrm{x}$ est-il venu ?

$\mathrm{b}$ '.pour quels $\mathrm{x}$ est-il vrai que $\mathrm{x}$ sont venus et quand sont-ils venus?

c. Přišel Martin, a to včera.

(est) venu Martin, et cela hier

c'.Přrišel Martin, Jan a Petr, a přšli včera.

(est) venu Martin, Jean et Pierre, et (ils sont) venus hier

(8)
a. Kdo
přišel a kdy?
$(8 \mathrm{a}=8 \mathrm{~b})$
qui-NOM (est) venu et quand
b. Kdo prišel a kdy
qui-NOM (est) venu et quand (est-il) venu ${ }_{6}^{6}$ L'ordre des interrogatifs dans les questions multiples en tchèque est libre et indique
l'ordre des mots dans la réponse. 


\section{LA FONCTION SYNTAXIQUE DES INTERROGATIFS}

L'acceptabilité des questions multiples et des questions avec coordination dépend de la fonction des interrogatifs dans la phrase (arguments, circonstants).

\subsection{Les interrogatifs argumentaux}

Lorsque les interrogatifs représentent le sujet ('qui-NOM') et l'objet direct ('quiACC') d'un même verbe, les questions multiples sont acceptables, cf. (9). Les questions avec coordination de type 1 sont également acceptables, cf. (10) ${ }^{7}$. En revanche, les questions avec coordination de type 2 sont exclues, cf. (11).

(9)
a. Kdo koho doporučil
komisi ?
qui-NOM qui-AC
a) recommandé (à la) commission
b. Koho kdo doporučil
komisi ?
qui-ACC qui-NOM (a) recommandé (à la) commission

(Q multiple)

(10)
a. Kdo a koho
doporučil
komisi ?

qui-NOM et qui-ACC (a) recommandé (à la) commission

b. Koho a kdo doporučil komisi ? qui-ACC et qui-NOM (a) recommandé (à la) commission

(coord. de type 1)

(11)
a.*Kdo
doporučil
komisi
a koho?
qui-NOM (a) recommandé (à la) commission et qui-ACC
b. * Koho doporučil
komisi
a kdo ?
qui-ACC (a) recommandé (à la) commission et qui-NOM

(coord. de type 2)

L'acceptabilité reste la même lorsque les interrogatifs représentent deux compléments d'un verbe (objets direct et indirect), cf. (12), (13) et (14). Les questions avec coordination de type 2 peuvent devenir acceptables seulement si l'interrogatif final est un complément facultatif, comme l'objet indirect en (14b).

(12)
a. Komu co
co řekl ?
qui-DAT quoi-ACC (a-t-il) dit
b. Co komu řekl ?

quoi-ACC qui-DAT (a-t-il) dit

(13) a. Komu a co řekl ? qui-DAT et quoi-ACC (a-t-il) dit

b. Co a komu řekl ?

quoi-ACC et qui-DAT (a-t-il) dit

(coord. de type 1)

(14) a. *Komu řekl a co ? qui-DAT (a-t-il) dit et quoi-ACC
b. $\mathrm{Co}$
řekl a komu?

quoi-ACC (a-t-il) dit et qui-DAT

(Q multiple)

(coord. de type 2)

\footnotetext{
${ }^{7}$ On notera qu'il est possible d'accentuer chacun des interrogatifs coordonnés.
} 


\subsection{Les interrogatifs circonstants / ajouts}

Lorsque les deux interrogatifs sont des circonstants ou des ajouts ('quand', 'comment'), les questions multiples sont agrammaticales, cf. (15). Les questions avec coordination sont, en revanche, acceptables, cf. (16) et (17). Nous reviendrons sur l'exemple (15) dans la section 5.1.

(15)
a.*Kdy jak
skončila válka $\mathrm{v}$ Bosně ?
b.*Jak
quand commen
nent (a) fini
guerre en Bosnie
fincila válka $v$ Bosně
comment quand (a) fini guerre en Bosnie

(Q multiple)

(16) a. Kdy a jak skončila válka $\mathrm{v}$ Bosně ?

(coord. de type 1)

quand et comment (a) fini guerre en Bosnie
b. Jak a kdy skončila válka v Bosně ? comment et quand (a) fini guerre en Bosnie

(17) a. Kdy skončila válka v Bosně a jak ? quand (a) fini guerre en Bosnie et comment
b. Jak skončila válka v Bosně a kdy?
comment (a) fini guerre en Bosnie et quand

(coord. de type 2)

\subsection{Les interrogatifs mixtes}

Les questions multiples qui comportent deux interrogatifs de type différent sont difficilement acceptables avec 'comment' et 'pourquoi', cf. (18) vs. (19). qui-ACC où / quand es (tu) vu où / quand qui-ACC es (tu) vu
(18) a. Koho kde/kdy jsi viděl?
b. Kde / kdy koho jsi viděl ?
(19) a.??Kdo jak / proč hodnotil houslisty ?

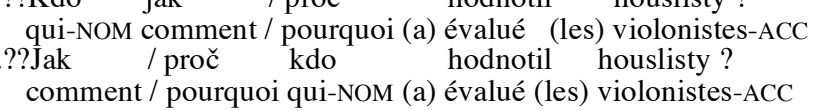

De même, les questions avec coordination de type 1 sont légèrement dégradées avec 'comment' et 'pourquoi' en position initiale, cf. (21).

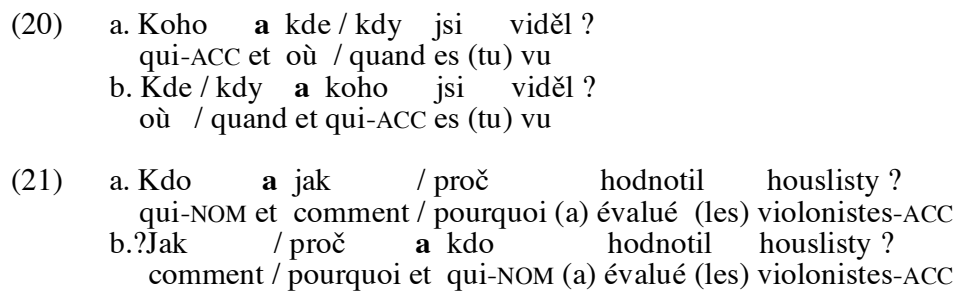


Le contraste entre (21a) et (21b) relève d'une contrainte pragmatique. Il est plus facile d'interroger l'individu et de questionner ensuite le paramètre de manière caractérisant la propriété $\mathrm{P}$ qui s'applique à cet individu que l'inverse. Le paramètre de manière questionné en $(21 \mathrm{~b})$ présuppose en effet l'existence d'un individu qu'il est donc pragmatiquement difficile de questionner par la suite.

En ce qui concerne le contraste entre (18) et (19), nous proposerons de l'expliquer par le caractère (non-)quantifiable des interrogatifs dans la section 5.1 .

Enfin, les questions avec coordination de type 2 sont exclues lorsque l'interrogatif final est un argument obligatoire du verbe, cf. (22b) et (23b).

(22)
a. Koho
potkal a kde/kdy?
qui-ACC (a-t-il) rencontré et où / quand
b. ${ }^{*}$ Kde / kdy potkal a koho?

où / quand (a-t-il) rencontré et qui-ACC

(23) a. Kdo hodnotil houslisty a jak / proč ?

qui-NOM (a) évalué (les) violonistes-ACC et comment / pourquoi

b.*Jak / proč hodnotil houslisty a kdo ?

comment / pourquoi (a-t-il) évalué (les) violonistes-ACC et qui-NOM

\subsection{Récapitulatif}

Le tableau suivant résume l'acceptabilité des questions multiples et des questions avec coordination varie selon le type d'interrogatifs ${ }^{8}$.

\begin{tabular}{|c|c|c|c|}
\hline Interrogatifs & Q multiples & Coordination 1 & Coordination 2 \\
\hline qui quoi & kdo co / co kdo & kdo a co / co a kdo & $* \mathrm{kdo} \ldots$ a co $/ * \mathrm{co} \ldots$ a kdo \\
\hline quoi à-qui & co komu / komu co & co a komu / komu a co & ${ }^{(*)}$ co ... a komu / *komu ... a co \\
\hline qui quand & kdo kdy / kdy kdo & kdo a kdy / kdy a kdo & 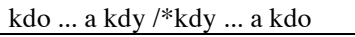 \\
\hline qui comment & ??kdo jak /??jak kdo & kdo a jak / ?jak a kdo & kdo ... a jak / *jak ... a kdo \\
\hline quand comment & *kdy jak /*jak kdy & kdy a jak / ?jak a kdy & kdy ... a jak / jak ... a kdy \\
\hline
\end{tabular}

\section{L'APPARENCE DE LA PARATAXE DANS LES QUESTIONS MULTIPLES}

Les données observées dans la section 3 suggèrent que les questions multiples et les questions avec coordination de type 1 ne diffèrent pas uniquement par la réalisation phonétique d'une conjonction. En effet, si c'était le cas, nous ne pourrions pas expliquer pourquoi l'acceptabilité de ces questions varie bien que les interrogatifs présents sont identiques.

En nous appuyant sur des phrases qui comportent l'auxiliaire 'être', nous pouvons cependant montrer que la parataxe des interrogatifs dans les questions

\footnotetext{
${ }^{8}$ Dans ce tableau, 'quand' peut être remplacé par 'où' et 'comment' par 'pourquoi'.
} 
multiples n'est qu'apparente. L'auxiliaire 'être' en tchèque est un clitique qui doit occuper la seconde position dans la phrase. La phrase (24) est en effet acceptable lorsque le clitique suit le premier interrogatif ${ }^{9}$. En revanche, la phrase (25) n'est acceptable que si le clitique suit les deux interrogatifs coordonnés. La position du clitique suggère donc que les interrogatifs préposés forment deux constituants, tandis que les interrogatifs coordonnés forment un seul constituant. ${ }^{10}$

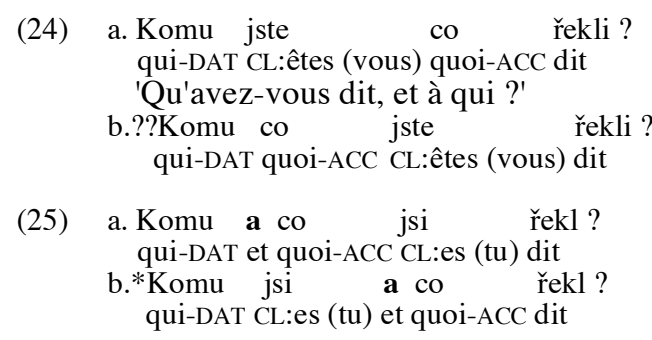

En outre, seuls les interrogatifs préposés peuvent être séparés par la conjonction de subordination (complémenteur) že ('que') ${ }^{11}$ qui précède d'éventuels clitiques, cf. (26a) contre (26b). L'impossibilité d'insérer že entre les interrogatifs en (26b) montre de nouveau que les interrogatifs coordonnés forment un seul constituant.
a. Kdo že
(mu) co
řek1 ?
qui-NOM que-COMP (CL:lui) quoi-ACC (a) dit
b. ${ }^{*} \mathrm{Kdo} \quad \check{\mathrm{e}} \quad(\mathrm{mu})$ a co řekl ? qui-NOM que- COMP (CL:lui) et quoi-ACC dit

La présence de la conjonction de subordination že n'indique pas que le deuxième interrogatif est subordonné au premier. Plutôt, že marque la frontière entre un élément distributeur (l'interrogatif qui le précède) et un élément distribué (l'interrogatif qui le suit) (voir l'analyse sémantique de la section 5.1).

Nous concluons que les questions multiples et les questions avec coordination de type 1 n'ont pas la même structure syntaxique. Autrement dit, si les interrogatifs coordonnés forment une structure coordonnée avec une conjonction visible, les interrogatifs multiples ne forment pas une structure coordonnée sans conjonction visible (c'est-à-dire une structure paratactique).

${ }^{9}$ Dans certains cas, le clitique pronominal réfléchi peut être placé après les deux interrogatifs préposés (cf. Lenertová 2001).

${ }^{10}$ Ceci est également valable pour les interrogatifs ayant la même fonction :

(i) Koho a čeho jste se báli?

qui-GEN et quoi-GEN CL:êtes (vous) CL:REFL craint = 'Qui et quoi avez-vous craint?'

(ii) *Koho jste se a čeho báli ?

qui-GEN CL:êtes (vous) CL:REFL et quoi-GEN craint

${ }^{11}$ En français familier, la conjonction 'que' peut aussi apparaître après un interrogatif :

(i) Où que tu vas? (ii) Qui que t'as cherché ?). 


\section{L'ANALYSE SYNTAXIQUE DES QUESTIONS A DEUX INTERROGATIFS}

\subsection{La structure des questions multiples}

Nous présentons une analyse dans le cadre du modèle Principes et Paramètres (Chomsky 1981 et 1986). Dans une question simple, le mot interrogatif est extrait de sa position de base (argument, circonstant) vers la périphérie gauche de la phrase interrogative $(=$ le domaine $\mathrm{CP})$. La présence de plusieurs interrogatifs à la périphérie gauche des questions multiples suggère que le $\mathrm{CP}$ comporte plusieurs positions susceptibles d'accueillir ces interrogatifs.

Selon l'hypothèse de Rizzi (1997), le domaine CP comporte en effet plusieurs projections destinées à accueillir les complémenteurs, les éléments topicalisés, les éléments focalisés et les interrogatifs. En adoptant cette hypothèse, nous proposons que le premier interrogatif occupe une position (spécifieur de ForceP) qui précède le complémenteur že ('que' = tête de ForceP), tandis que le deuxième interrogatif occupe une position qui suit le complémenteur (spécifieur de FinP), cf. (27a). Etant donné que les clitiques peuvent précéder ou suivre le deuxième interrogatif, celui-ci peut se trouver également dans une position plus bas que les clitiques. En admettant que les clitiques occupent la position la plus basse dans la périphérie gauche (= tête de FinP, cf. Lenertová 2001), l'interrogatif final serait dans ce cas adjoint à la phrase principale $(=\mathrm{TP})$ au-dessous de $\mathrm{CP}$, conformément à l'analyse de Rudin (1988), cf. (27b) .

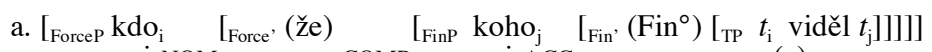

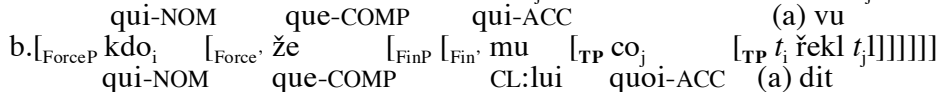

En admettant que la position syntaxique des expressions quantifiées reflète leur portée sémantique (cf. Beghelli \& Stowell (1997)), nous pouvons expliquer l'interprétation de liste de paires des questions multiples. Dans ces questions, l'un des interrogatifs se trouve hiérarchiquement plus haut dans la périphérie gauche de la phrase interrogative que l'autre. L'interrogatif situé plus haut porte (ou distribue) ainsi sur celui qui est situé plus bas. Nous obtenons par conséquent une lecture distributive (quantifiée), dans laquelle chaque élément du premier ensemble (dénoté par le premier interrogatif) est apparié à un élément du deuxième ensemble (dénoté par le deuxième interrogatif).

Comment expliquer alors l'agrammaticalité des questions avec deux interrogatifs circonstants en (28b) et le contraste entre (28b) et $(28 c)^{12}$ ?

(28)
a. Kde / Jak ho
uhodil ?
(Q simple)
où / comment CL:le (a-t-il) frappé
b.*Kde jak ho uhodil ?
(Q multiple, cf.(15))

où comment CL:le (a-t-il) frappé

${ }^{12}$ Les interrogatifs 'où' a 'comment' peuvent être interprétés de deux manières différentes 'où' = à quel endroit / sur quelle partie du corps, 'comment' = de quelle manière / à quel degré) mais ceci n'a pas d'influence sur le contraste entre (28a) et (28b). 
c. Kde a jak ho ahodil ?
où et comment CL:le (a-t-il) frappé

Les interrogatifs 'où', 'qui', 'quoi' et 'quand' sont quantifiables et individualisables. Ils peuvent donc distribuer les uns sur les autres. En revanche, 'comment' et 'pourquoi' ne sont pas quantifiables, ou, plutôt, ne permettent pas d'individuation. Ils ne peuvent donc entrer dans une relation entre un élément distributeur et un élément distribué. La phrase (28a) est inacceptable, car jak ne peut pas distribuer sur kde (et vice versa). En revanche, si nous ajoutons un interrogatif quantifiable à la question (28a), celle-ci devient tout à fait acceptable, cf. $(29)^{13}$.

Les interrogatifs coordonnés en (28b) forment, contrairement aux interrogatifs multiples, un seul constituant. L'interprétation quantifiée du premier interrogatif par rapport au deuxième est donc exclue; la coordination de type I reçoit en effet une interprétation spécifique. Par conséquent, la phrase (28b) est grammaticale au même titre que les questions simples en (28c) le sont.

\subsection{La structure des questions avec coordination}

Selon les travaux de Banréti (1992) sur le hongrois, les questions avec coordination sont des coordinations de deux phrases. Chaque phrase contient un mot interrogatif et l'une d'elles subit l'effacement de tous ses constituants sauf l'interrogatif. Dans la coordination de type 1, l'effacement se produit dans le premier conjoint, cf. (30a). Dans la coordination de type 2, l'effacement se produit dans le deuxième conjoint, $\mathrm{cf}$. (30b).
(30)
a. [[Kdo
b. [[Kdo
přišel] $\mathbf{a}[\mathrm{kdy}$
přišel] a [kdy
přišel]]
prišsel]
(coord. de type 1)
(coord. de type 2)

L'analyse en (30) est cependant problématique pour trois raisons :

1. Les interrogatifs en (30a) ne forment pas un constituant. Les questions comportant les clitiques et la conjonction de subordination dans la section 4 ont cependant montré que les interrogatifs dans la coordination de type 1 forment un constituant.

2. Les deux types de coordination ont la même structure en Forme logique (cf. Collins 1997). Elles sont donc supposées être équivalentes du point de vue sémantique. Mais cette équivalence ne permet pas d'expliquer le fait que la ${ }^{13}$ Notons qu'en (29), le clitique ho ('le') s'intercale entre les interrogatifs quantifiables d'un
côté et l'interrogatif 'comment' de l'autre. 
coordination de type 1 en (31a) est acceptable, alors que la coordination de type 2 avec les mêmes interrogatifs en (31b) ne l'est pas.

(31)
a. Kdo
a koho
uhodil ?
qui-NOM et qui-ACC (a) frappé
b. *Kdo uhodil a koho ?
qui-NOM (a) frappé et qui-ACC

3. En admettant que tous les arguments du verbe sont présents dans la structure de la coordination en (30a), l'analyse phrastique doit supposer que l'objet direct dans la première phrase est une trace et le sujet dans la deuxième phrase un pronom nul, cf. (32). Or, la trace de l'objet en (32) n'est pas liée, et le sujet nul, qui est référentiellement défini, n'admet pas d'être questionné.

$$
\begin{aligned}
& \text { *[Kdo uhodil } \left.\mathrm{t}_{\mathrm{obj}}\right] \\
& \text { qui-NOM (a) frappé (qui-OBJ) et qui-ACC }
\end{aligned}
$$

Nous concluons que l'analyse phrastique n'est pas appropriée pour la coordination de type 1 (que nous analyserons dans la section 5.4), mais seulement pour la coordination de type 2 (cf. section 5.3).

\subsection{La structure de la coordination de type 2}

Nous avons vu que dans la coordination de type 2, le deuxième interrogatif ne peut pas être un argument du verbe de la phrase interrogative. C'est un élément facultatif qui peut être considéré comme externe à cette phrase. L'analyse phrastique de Banréti (1992) permet de capter ces propriétés. La phrase qui comporte le premier interrogatif est complète et son acceptabilité n'est pas altérée par l'ellipse dans la phrase qui comporte le deuxième interrogatif. En outre, si les questions avec coordination de type 2 contiennent deux questions simples, nous expliquons l'interprétation de paire spécifique des interrogatifs dans ces questions. Les questions avec coordination de type 2 sont en effet toujours interprétées comme des coordinations de deux phrases (cf. section 2).

Par ailleurs, on peut penser que la phrase elliptique (la séquence Conj Int $_{2}$ ) n'est pas coordonnée avec la phrase qui précède, mais qu'elle est adjointe à cette phrase. Trois arguments étayent une analyse comme ajout :

1. La séquence Conj Int $_{2}$ peut être prosodiquement décrochée de la phrase interrogative, comme en (33a).

2. La séquence décrochée est mobile, c'est-à-dire qu'elle peut apparaître à l'intérieur de la phrase interrogative, comme en (33b). Or, la mobilité est une propriété typique des constituants adjoints.

3. La phrase interrogative peut comporter plusieurs interrogatifs. La séquence Conj Int $_{2}$ peut s'adjoindre à une question simple aussi bien qu'à une question multiple, cf. (33c).

$$
\begin{aligned}
& \text { a. Kdy že jsi potkal Jana, - A KDE ? } \\
& \text { quand que CL:as (tu) rencontré Jean-ACC, et où }
\end{aligned}
$$




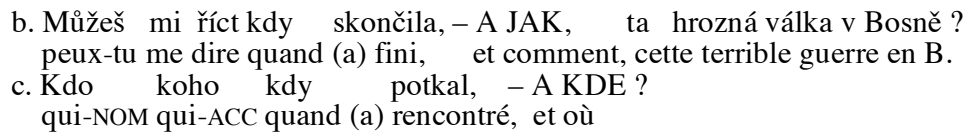

\subsection{La structure de la coordination de type 1}

Nous avons montré plus haut que la coordination de type 1 correspond à un seul constituant. Il ne s'agit pas donc d'une coordination phrastique, mais d'une coordination syntagmatique. Les deux interrogatifs sont en effet interprétés comme appartenant à une seule et même phrase. Nous proposons que les interrogatifs coordonnés forment un syntagme coordonné (CoP, cf. Lipták (2001)). Nous admettons par ailleurs que la conjonction est la tête de ce syntagme (cf. Johannessen (1998)), comme en (34a). Ce syntagme se trouve à la périphérie gauche de la phrase interrogative de même que les syntagmes interrogatifs dans les questions simples et multiples, cf. (34b).

(34) a. $\left[{ }_{\mathrm{CoP}}\left[\right.\right.$ Conjoint $\left._{1}\right]\left[\mathrm{Co}^{\prime}\right.$ Conj $^{\circ}\left[\right.$ Conjoint $\left.\left._{2}\right]\right]$

b. $\left[\right.$ Forcep $\left.\left[\mathrm{CoP} \operatorname{Int}_{1}\left[\mathrm{Conj}^{\circ} \operatorname{Int}_{2}\right]\right]\left[\mathrm{FinP}_{\mathrm{TP}}[\ldots .].\right]\right]$ ?

Dans une coordination syntagmatique, les constituants coordonnés ne peuvent pas en général être de catégories et/ou de fonctions différentes, cf. (35a). Notre analyse prévoit donc que la coordination des interrogatifs ayant la même fonction grammaticale est possible (cf. exemple (3b) plus haut), mais pas la coordination des interrogatifs ayant des fonctions différentes, comme en (35b). Comment les coordinations comme celle en (35b) sont-elles donc autorisées ?

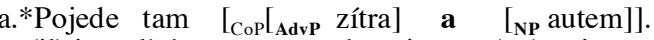

$$
\begin{aligned}
& \text { (il) ira là-bas demain et (en) voiture-INSTR } \\
& \text { b. }\left[_{\mathrm{CoP}}\left[\mathrm{Int}_{\mathrm{nt}} \mathrm{Kdy}\right]\right. \text { a [ [nt jak]] tam pojede ? } \\
& \text { quand et comment là-bas (il) ira }
\end{aligned}
$$

Selon Lipák (2001), la coordination des constituants de fonctions différentes est possible à condition qu'ils soient focalisés ${ }^{14}$. L'exemple (35a) devient en effet acceptable lorsque les conjoints ont le statut de focus informationnel, comme l'indique les majuscules en (36a). La phrase (36a) est appropriée par exemple lorsqu'elle constitue la réponse à la question $(35 \mathrm{~b})$.

Il est généralement admis que les constituants interrogatifs constituent la partie focalisée de l'énoncé. Nous expliquons ainsi que les interrogatifs puissent être coordonnés même s'ils n'ont pas la même fonction grammaticale, car ils ont la même fonction de focus sur le plan informationnel, cf. (36b).

\footnotetext{
${ }^{14}$ (i) John met *[Mary and in her house] / [MARY, and in HER house]. (anglais)

(ii) Ide *[mindenki és mindig] / [MINDENKI és MINDIG] be-jöhet. (hongrois) ici chacun et toujours / chacun et toujours PART-peut-entrer 'Chacun peut entrer ici et c'est valable pour toujours.'
} 
La même analyse pourrait s'appliquer à des quantifieurs de type 'qui que ce soit', qui reçoivent une interprétation de focus informationnel, et qui peuvent en effet être coordonnés, $\mathrm{cf}$. (36c) ${ }^{15}$.

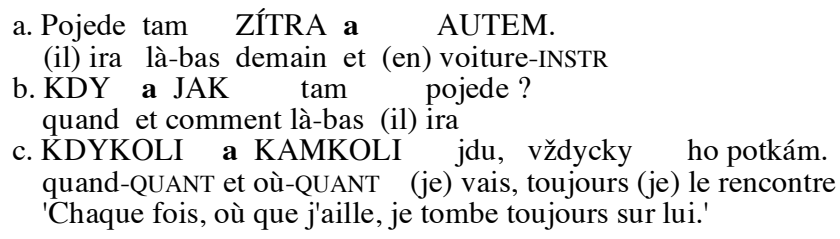

L'analyse syntagmatique proposée apparaît appropriée pour la coordination de type I. Elle pose néanmoins un problème général du point de vue de l'extraction. Le syntagme de coordination interrogatif existe en effet en tant que constituant extrait, mais pas en tant que constituant non-extrait. Autrement dit, le syntagme interrogatifs extrait ne peut être associée à aucun constituant in situ dans la phrase, contrairement à ce qu'on observe en général dans les structures à extraction ${ }^{16}$. Ce problème dépasse cependant le cadre de cet article et sera traité dans nos travaux ultérieurs.

\section{CONCLUSION}

Dans cet article, nous avons analysé trois types de questions (Qs) à deux interrogatifs en tchèque : Qs multiples $\left(\right.$ Int $_{1}$ Int $_{2} \ldots$...? $)$, Qs avec coordination de type 1 ( Int $_{1}$ Conj Int $_{2} \ldots$ ?) et Qs coordination de type 2 (Int $\ldots$ Conj Int I $_{2}$ ?). Nous avons montré que ces questions diffèrent sur le plan sémantique (interprétation) aussi bien que sur le plan syntaxique (fonction grammaticale des interrogatifs) et qu'elles ne peuvent pas donc avoir la même structure syntaxique. Nous avons présenté en particulier des arguments (relativement indépendants d'un cadre particulier) contre une analyse paratactique des questions multiples. Les interrogatifs dans ces constructions sont des constituants indépendants qui occupent chacun une position associée à une interprétation quantifiée dans la périphérie gauche de la phrase. Au contraire, les interrogatifs coordonnés du type 1 forment un syntagme coordonné qui occupe une seule position dans la périphérie gauche de la phrase. Nous avons également défendu l'idée que, malgré leurs fonctions grammaticales différentes, les interrogatifs peuvent être coordonnés, car ils ont la même fonction de focus sur le plan informationnel.

Enfin, nous avons montré que la séquence Conj Int dans les coordinations de type 2 doit être analysée comme une phrase elliptique. On peut considérer que cette phrase est coordonnée à la phrase qui précède, ou alors adjointe.

15 Notons que ces quantifieurs sont des formes composées qui comportent les interrogatifs: $k d o k o l i=$ ' qui que ce soit', $k d e k o l i=$ 'où que ce soit', etc..

${ }^{16}$ D'autres constructions posent le même problème, par exemple la topicalisation des complétives en anglais (Bresnan 1972) ou celle des SV incomplets en allemand (Haider 1990 parmi d'autres). 